Trivent Publishing

(C) The Authors, 2016

Available online at http://trivent-publishing.eu/

Engineering and Industry Series

Volume Power Systems, Energy Markets and Renewable Energy Sources in

South-Eastern Europe

\title{
Spectral Performance of PV Modules of Different Technologies
}

\author{
Fotis Mavromatakis, ${ }^{1}$ Frank Vignola ${ }^{2}$ \\ ${ }^{1}$ Department of Electrical Engineering, TEI of Crete, Greece \\ ${ }^{2}$ Department of Physics, University of Oregon, USA, fev@uoregon.edu
}

\begin{abstract}
The power produced by a photovoltaic array depends, among other factors, upon the spectral responsivity of the modules and the spectral distribution of the irradiance at the plane of the array. The spectral responsivity is related to the type of solar cell used for the module. The spectral composition of the incident irradiance onto the module depends on the atmospheric conditions, the path length of the solar irradiance through the atmosphere and the spectral characteristics of the ground and diffuse components. Data acquired by the Aerosol Robotic Network are employed to examine the variability of aerosols and water vapor at selected sites. A software spectral model is used to explore the effects of these atmospheric constituents upon a spectral correction factor. The analysis found that the water vapor content of the atmosphere affects the spectral correction factor more than the aerosol optical depth.
\end{abstract}

\section{Keywords}

Photovoltaic technology; spectrum; spectral response; air mass; modelling 


\section{Introduction}

Spectral corrections need to be applied in cases where cells are used as sensors of solar irradiance and when software models are used to predict PV system performance. The spectral (correction) factor or spectral mismatch correction results from the mismatch between the incident spectral irradiance distribution and the reference AM1.5 spectrum and the characteristics of the spectral correction factor depend on the given PV module technology. The major parameters that determine the shape of the reference AM1.5 solar spectrum is the Angstrom turbidity at $500 \mathrm{~nm}$ which is set to 0.084 and the total column of water vapour (precipitable water) which is set to $1.42 \mathrm{~cm}$. Almost twenty years ago King1 et al proposed a methodology to determine the changes in spectral performance as a function of the path that light traverses through the atmosphere (airmass). It was assumed that spectral variations are a function of airmass alone and that the variations due to aerosols and water vapor content are less important. The King methodology consists of measurements of the short circuit current of a PV cell or module along with measurements of the incoming solar irradiance as the sun changes position on the celestial sphere. The module is always held perpendicular to the solar rays to avoid effects introduced by the varying angle of incidence while the short circuit current is adjusted for module temperature. In the analysis, the spectral correction factor is evaluated using the ratio of the normalized short circuit current (Isc/Isc_STC) over the normalized irradiance (Gt/GSTC). Higher values suggest better performance than the standard test conditions while lower values suggest poorer performance. Typically, crystalline modules perform better at air masses higher than 1.5 while amorphous PV modules, given their narrower spectral response, perform worse with respect to STC in the same range. However, under real atmospheric conditions neither the aerosol optical depth nor the water vapor content are equal or close to the values adopted to represent the AM1.5 solar spectrum. Consequently, it is interesting to explore the spectral variations introduced by the different conditions found throughout a year and at different sites. 


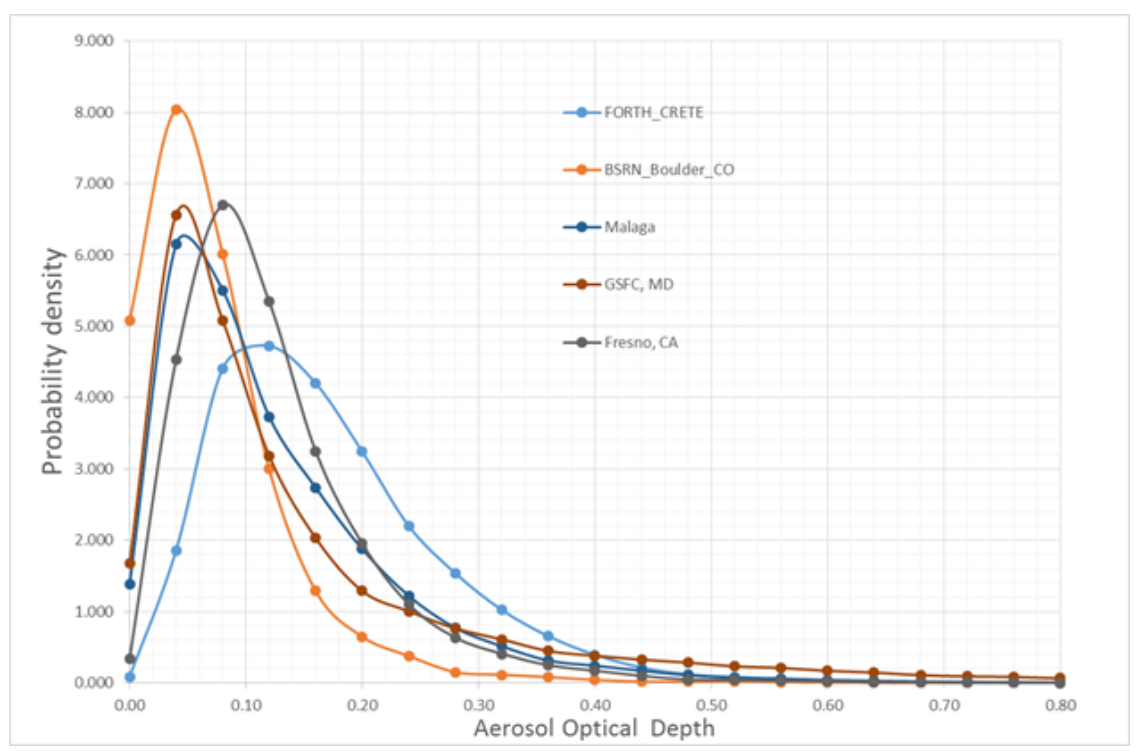

Fig. 1 Probability density of the Aerosol Optical Depth

\section{Experimental and model data}

In order to study such variations we employ data from the Aerosol Robotic Network2 (AERONET) experiment. The AERONET records aerosol optical depths at ground level and at different wavelengths along with the water vapor content of the atmosphere. The data are subject to filtering procedures in order to validate their quality. In this work we only use Level 2.0 data that represent the best quality. The currently selected sites are FORTH Crete, BSRN Boulder CO, Malaga Spain, GSFC MD, and Fresno CA., USA. For these sites, the monthly averages of aerosol optical depth at $500 \mathrm{~nm}(\beta)$ and precipitable water (pw) are calculated along with the standard deviation and the minimum and maximum recorded values. In addition, the distribution of $\beta$ and pw between winter and summer are also calculated to identify seasonal effects. It is possible to study seasonal effects in more detail, however, for this initial examination we simply separate a year in two parts, roughly the times between the equinoxes. Winter time is considered the time from October to March, while summer is considered the time from April to September. Fig. 1 and 2 show the distribution (probability density) of the aerosol optical depth and precipitable water for the referenced cities and have been deduced using all available Level 2.0 data from the ARONET site. The variations seen among the various sites suggest that the use of a single expression may not suffice for the proper calculation of spectral corrections. Variations between winter and summer are also observed (seasonal 
variations). This implies that the spectral correction factor will also vary. Fig. 3 shows the frequency histograms for the Munich AERONET station for winter and summer. Approximately 30000 data points designated as Level 2.0 are used to create these histograms. In general, seasonal effects are stronger in the water vapor content of the atmosphere than in the aerosol optical depth. Intraday variations are also observed in the AERONET measurements. Both of the above quantities are input to the SMARTS5 code in order to calculate the synthetic solar spectra. Given the observed variations is aerosol optical depth and precipitable water the simulated solar spectra cover the range from 0.054 to 0.604 (step of 0.01 ) and from $0.5 \mathrm{~cm}$ to $4.5 \mathrm{~cm}$ (step of $0.1 \mathrm{~cm}$ ), respectively, while the air mass ranges from 1.0 to 6.0 in steps of 0.1 .

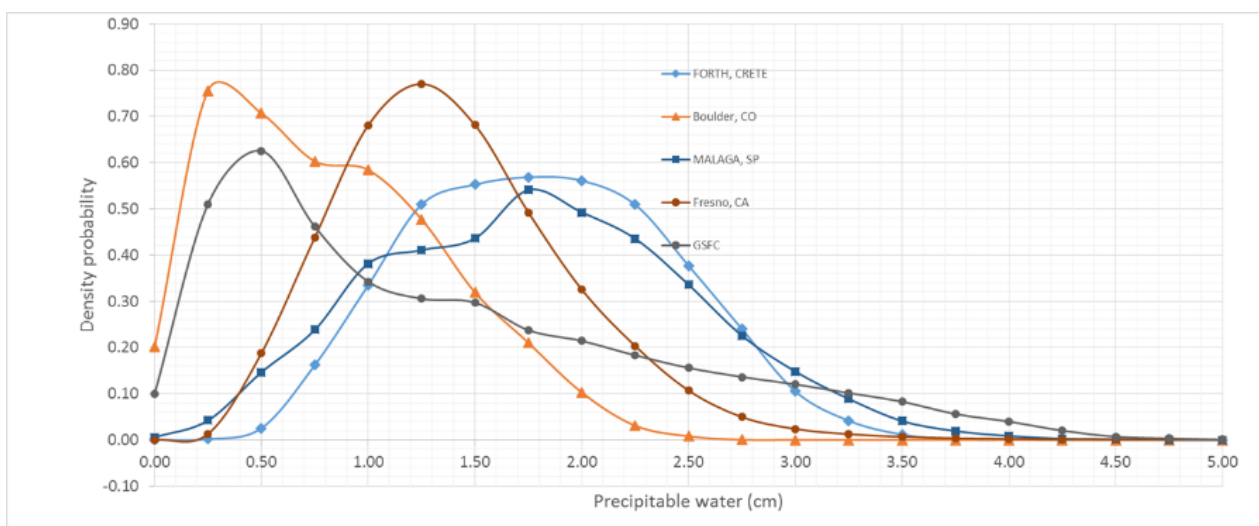

Fig. 2 The probability density distribution of the water vapour content
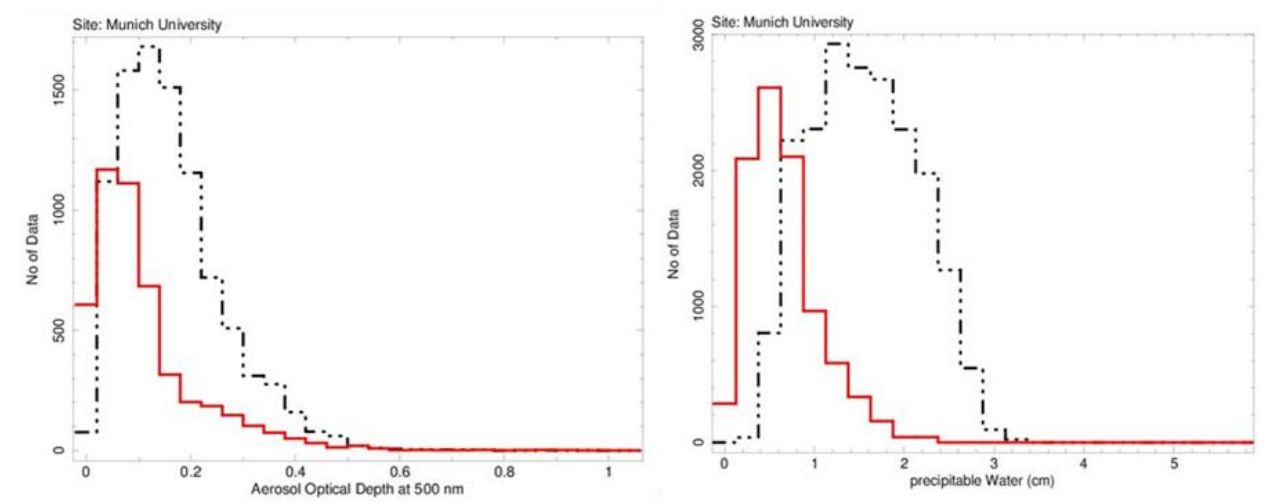

Fig. 3 The frequency histograms of aerosol optical depth and precipitable water for winter (red curve) and summer (dash-dotted) 
The resulting solar spectra are convolved with the responsivity of a solar cell (e.g. mono, multi crystalline, CdTe, etc.) and with the response of a pyranometer to calculate the spectral correction factor of the AOD. Fig. 4 shows the spectral correction factor assuming a typical multi-crystalline response and adopting two different values for the aerosol optical depth $(0.084$ and 0.150$)$. These last two values were selected to allow the reader to visualize the effect

\section{Discussion}

The data show that for any given airmass and a given aerosol optical depth, the spectral correction factor is an increasing function of water vapour content. The dependence on aerosol optical depth is not as strong as on the water vapour content. Note also the different scale on the y-axis and the fact that a-Si technology modules are less affected at higher air masses by the different aerosol optical depths. Similar plots like those in Fig. 4 have been created for specific values of the water vapour content and for the full range of AODs. It turns out that the effect of the AOD on the spectral correction factor is stronger at higher air masses compared to lower air masses. The effect is of the order of $1 \%$ around an airmass of 1.5, while at an airmass of 2.0 the spread in the spectral correction factor is around 3\%. Modules of other technologies are also used in the simulation process and the results are currently under study. It is evident that the spectral correction factor is a function of three parameters (air mass, water vapor content and aerosol optical depth). The current analysis focuses on the spectral quality of the global irradiance. The above analysis shows that a simple polynomial expression of the spectral correction factor is not sufficient to account for the various conditions under which photovoltaic systems operate. More complicated expressions are required to accommodate the effects introduced by precipitable water and aerosols. The simulated data re fit with mathematical expressions. We also employed data from the NREL6 experiment where modules of different technologies were deployed at three different US sites for more than one year. Unfortunately, nearby AERONET data are available at only one site (Golden, CO). Fig. 5 shows essentially the spectral correction factor for a multicrystalline module and for two different time intervals where the precipitable water differ significantly. The aerosol optical depth at $500 \mathrm{~nm}$ is similar between these two time interlvals. Despite the fact that the data span over several days, a trend may be seen in this data. The effects of the beam and diffuse components on the spectral correction factor will also be examined after the thorough investigation of the global irradiance. Work is 
currently being done in establishing expressions for the spectral correction factor for a number of different photovoltaic technologies.
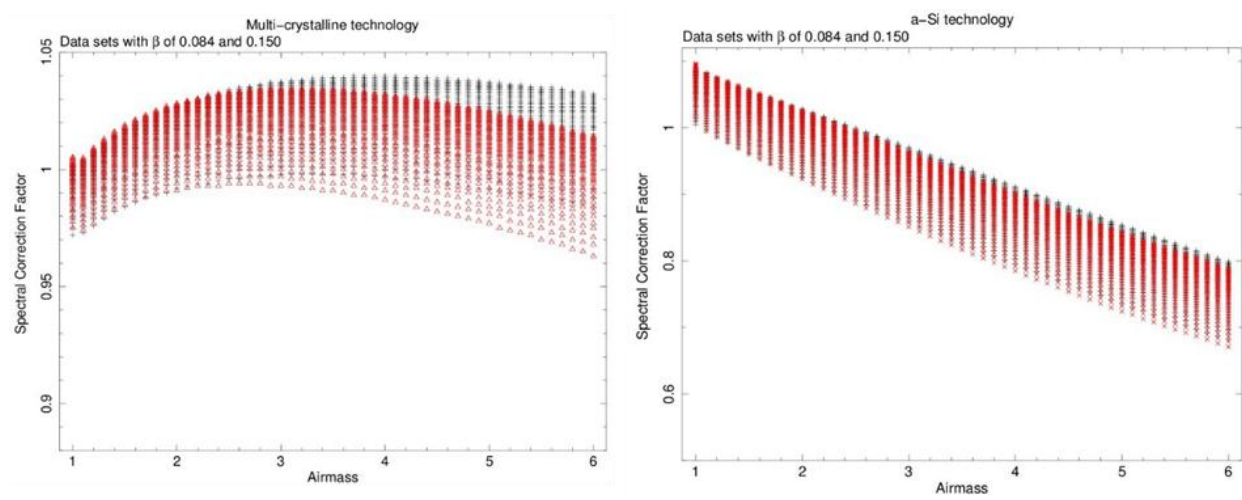

Fig. 4 The effect of water vapor content on the spectral correction for two different aerosol optical depths (crosses mark $\beta=0.084$, triangles mark $\beta=0.150$ )

\section{Conclusions}

The spectral response of a photovoltaic module is an important characteristic related to its performance combined with the quality of the solar spectrum at the site of installation. Electromagnetic radiation from the sun interacts with the constituents of the atmosphere before reaching a pv module. Mainly software simulations show that the water vapor content of the atmosphere affects the spectral correction factor more than the aerosol optical depth. Experimental data are also being examined to verify the findings of this work.

\section{References}

King D. L., Kratochvil J. A., Boyson W.E., "Measuring Solar spectral and angle of incidence effects on photovoltaic modules and solar irradiance sensors", 26th IEEE Photovoltaic Specialists Conference, September 29-October 3, 1997, Anaheim, California.

Holben B.N., T.F. Eck, I. Slutsker, D. Tanré, J.P. Buis, A. Setzer, E. Vermote, J.A. Reagan, Y. Kaufman, T. Nakajima, F. Lavenu, I. Jankowiak, and A. Smirnov, 1998: “AERONET - A federated instrument network and data archive for aerosol characterization”, Rem. Sens. Environ., 66, 1-16. 
Lee M., Panchula A., "Combined air mass and precipitable water spectral correction for pv modelling", 4th PV Performance Modelling and Monitoring Workshop, 22-23 October, 2015, Cologne, Germany

Mavromatakis F., Vignola, F., "Spectral corrections for pv performance modelling", 4th PV Performance Modelling and Monitoring Workshop, 2223 October, 2015, Cologne, Germany

Gueymard, C. "Parameterized transmittance model for direct beam and circumsolar spectral irradiance", Solar Energy, Volume 71, Issue 5, November 2001, Pages 325-346.

Marion W., Anderberg A., Deline C., Glick S., Muller M., Perrin G., Rodriguez J., Rummel S., Terwilliger K., and Silverman T.J., "User's Manual for Data for Validating Models for PV Module Performance", NREL/TP-5200-61610, 2014 